\title{
NK Cells in HIV Disease
}

\author{
Eileen Scully $^{1,2} \cdot$ Galit Alter $^{1}$
}

Published online: 21 March 2016

(C) The Author(s) 2016. This article is published with open access at Springerlink.com

\begin{abstract}
Natural killer (NK) cells play a critical role in viral immunity. In the setting of HIV infection, epidemiologic and functional evidence support a role for NK cells in both protection from new infection and in viral control. Specifically, NK cells directly mediate immune pressure leading to virus evolution, and NK cell receptor genotypic profiles, clonal repertoires, and functional capacity have all been implicated in virus containment. In addition, indirect NK cell-mediated antibody-dependent cellular cytotoxicity has been linked to vaccine-induced protective immunity against HIV infection. With recent advances in our understanding of NK cell deficiency, development, memory-like responses, and editing of the adaptive immune system, the opportunities to direct and exploit NK cell antiviral immunity to target HIV have exponentially grown. In this review, we seek to highlight the intersections between discoveries in basic NK cell biology and the challenges of HIV chronic infection, vaccine development, and cure/eradication strategies.
\end{abstract}

This article is part of the Topical Collection on HIV Pathogenesis and Treatment

Galit Alter

galter@partners.org

Eileen Scully

escully@partners.org

1 Ragon Institute of Massachusetts General Hospital, Massachusetts Institute of Technology and Harvard, 400 Technology Square, Cambridge, MA 02139, USA

2 Division of Infectious Disease, Department of Medicine, Brigham and Women's Hospital, Boston, MA 02130, USA
Keywords HIV $\cdot$ Innate immunity $\cdot \mathrm{NK}$ cell .

Antibody-dependent cellular cytotoxicity $\cdot \mathrm{NK}$ cell memory . Natural killer (NK) · Viral immunity · HIV pathogenesis ·

Review

\section{Introduction}

Natural killer (NK) cells occupy a unique niche in the immune response, bridging the innate and adaptive immune systems. They are capable of recognizing generic signals of stress, transformation, or infection with immediate effector function. NK cells are the critical antiviral effectors of the innate immune system, and natural deficiencies are associated with susceptibility to viral infections [1,2]. However, there is still a nascent understanding of their pleiotropic functions as direct effectors, but also as editors of adaptive immunity in both infection and vaccination [3]. Recent work has also highlighted the adaptive features of NK cells that can confer a memorylike phenotype in the setting of infection [4]. NK cells have the potential to directly respond to viruses, to develop memorylike responses after initial pathogen encounter or vaccination, and to shape the adaptive immune response (Fig. 1).

As the understanding of NK cell biology grows, there are promising avenues to address several current challenges in HIV: development of an effective vaccine, control of residual inflammation in treated disease, and emerging strategies for eradication or cure. NK cell deficiency syndromes have revealed specific developmental programs that shape the mature NK cell population and can broadly modulate responses [5]. The HIV vaccine literature has identified the critical importance of antibody-dependent cellular cytotoxicicty (ADCC), mediated predominantly by NK cells, via non-neutralizing antibodies as a pathway to protective vaccine responses [6]. Importantly, these ADCC responses are associated with 


\section{DIRECT CYTOTOXICITY /}

CYTOKINE SECRETION

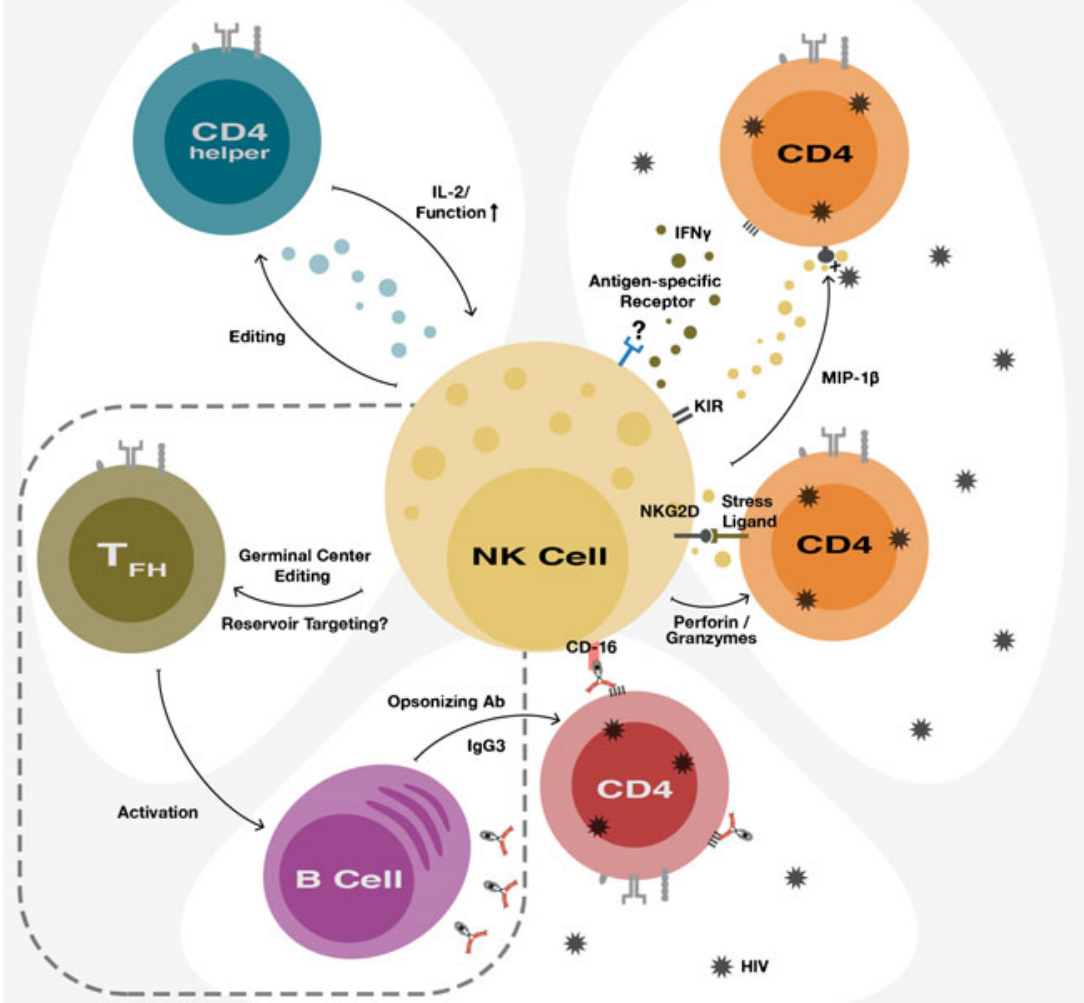

ANTIBODY-DEPENDENT CELLULAR CYTOTOXICITY

Fig. 1 Multiple facets of NK cell biology can be harnessed towards the goal of HIV prevention and eradication. (Right panel) NK cells can directly recognize HIV-infected targets through expression of stress ligands on infected cells or potentially through antigen-specific responses as has been demonstrated in the SIV infection in rhesus macaques. This recognition may lead to degranulation and target cell cytolysis. In addition, secretion of chemokines such as MIP-1 $\beta$ can block new rounds of infection and effectors such as IFN $\gamma$ can activate antiviral programs. (Bottom panel) NK cells can also be indirectly recruited to HIV-infected cells through CD16 engagement of the Fc receptor of antibodies bound to HIV epitopes. Optimization of these antibodies through subtypes with multiple coordinated activities and glycan patterns that direct maximal responses can enhance NK effector

specific immunoglobulin subclasses [7] and glycan modifications that directly impact the efficiency of NK cell responses $[8,9]$. This affords multiple avenues for optimization of antibodies to maximize NK cell function for preventative and therapeutic vaccination [10].

The profile of NK cell responsiveness beyond CD16mediated antibody recognition also points to anti-HIV function. High dimensional characterization of the combinatorial diversity of $\mathrm{NK}$ receptor expression offers new insights into the phenotypic features of protective NK cell responses and how they may be directed $[11,12 \bullet \bullet]$. Building on prior work showing antigen specificity in mouse models, recent studies have demonstrated NK cell function. (Left panel) NK cell cross-talk with the adaptive immune system also affords opportunities for modulating responses. NK cells edit $\mathrm{T}$ follicular helper $\left(\mathrm{T}_{\mathrm{FH}}\right)$ cells altering germinal center formation and antibody development through cross-talk with $\mathrm{B}$ cells. $\mathrm{T}_{\mathrm{FH}}$ cells are also a reservoir of HIV infection, and could potentially be targeted by NK cells in early infection or during curative interventions. NK cell editing of $\mathrm{CD}^{+} \mathrm{T}$ cell responses also contributes to immune exhaustion in chronic viral infection models, and limits immunopathology in acute infections that are cleared. There is the potential for bidirectional effects with NK cell limiting or enhancing CD4 function, and CD4 T cell cytokine production supporting improved $\mathrm{NK}$ cell effector capacity. In sum, there are multiple avenues through which NK cell function can be leveraged to optimize immune responses to HIV

memory responses to simian immunodeficiency virus (SIV) antigens in nonhuman primates, suggesting a pathway to induce antigen-driven memory in humans [13••]. In line with recent findings in the myeloid compartment delineating the phenomena of innate training and tolerance [14], unique epigenetic signatures in NK cells primed by cytomegalovirus (CMV) infection demonstrate how NK cells can be programmed by an initial pathogen (or potentially vaccine) exposure $[15 \bullet \bullet, 16 \bullet \cdot]$. Taken together, this work suggests infection can shape the NK cell repertoire and alter subsequent responses; a detailed understanding of this functional plasticity will inform efforts to develop preventative and therapeutic vaccines against HIV. 
In this review, we discuss the role of NK cells in the immune response to HIV in the context of recent advances in NK cell biology, highlighting opportunities to harness these innate cytolytic effector cells for the rapid containment and clearance of HIV.

\section{NK Cells in HIV Infection}

\section{The Role of NK Cells in the Immune Response to HIV Infection}

Population-level genetic associations between NK cell receptor expression and HIV-1 outcomes and evolution reveal the impact of NK cells on HIV-1 disease progression. The germline encoded inhibitory receptors including the killer immunoglobulin-like receptors (KIRs) heavily influence NK cell activation which is governed by the integration of activating and inhibitory signals. Interactions between KIRs and their cognate HLA ligands set a threshold for NK activity and have been shown to critically influence the course of viral infection, associating with resolution of hepatitis $\mathrm{C}$ infection [17]. In HIV, HLA/KIR combinations have been associated with the pace of disease progression $[18,19]$ and protection against disease acquisition $[20,21]$. The mechanisms conferring this protection may include both NK cell education/ licensing through inhibitory receptor ligation [22] and the direct interaction of KIRs with HIV-1-derived peptide motifs presented on HLA molecules. The latter is supported by virus evolution in the presence of specific KIRs; footprints in the HIV genome associate with KIR genotype. Specific viral variant/KIR combinations associate with differences in NK cell viral inhibition in vitro [23] and HLA/KIR combinations confer differences in HIV control [24]. The HLA/KIR interactions directed by specific HIV-derived peptides are further linked to measures of NK cell function in vitro and patterns of viral escape in population studies $[25,26]$. These data suggest that NK cell activation threshold, determined in part by the genetic array of inhibitory receptors, and the virus-derived peptides available for presentation on host HLA cooperate to define the protective efficacy of NK cell responses. The latter could be exploited to harness function; computation approaches may be able to predict peptides that modulate NK function based on host HLA/KIR patterns to individualize vaccine design.

These data demonstrate direct interactions between NK cells and HIV peptides; however, it is not known whether these signals govern in vivo effector function and which of the overlapping recognition pathways is sufficient for an effective response. Although counterintuitive, focusing on immune responses that HIV has evolved to evade may point to highly effective means of NK activation. HIV-mediated downregulation of HLA molecules, which shields infected cells from killing by $\mathrm{CD} 8^{+} \mathrm{T}$ cells [27], theoretically offers the "missing self" trigger for NK cells. However, this is limited by HIV immune evasion; the downregulation of HLA A and B by Nef is coupled to preservation of HLA C and E, maintaining self signals, and preventing NK activation [28]. Likewise, HIV infection directly triggers the upregulation of stress ligands for cytotoxicity receptors including NKG2D $[29,30]$. Again, HIV is able to limit expression of these ligands and other coactivating signals such as NTBA-4 through accessory proteins $[29,31]$. While the precise contribution of each activation pathway in natural infection is difficult to prove, from the perspective of prevention and cure, each could be leveraged to optimize responses. Small molecule inhibitors disabling immune evasion through viral accessory proteins may enhance native or vaccine-induced immune recognition.

ADCC is a potent means of NK cell control of HIV-1 infection. NK cells express the Fc $\gamma$ RIIIA receptor (CD16) that binds the constant $(\mathrm{Fc})$ domain of $\mathrm{IgG}$ antibodies. CD16 engagement is a strong activator of NK cell function, and allows antigen-specific recruitment of NK responses. Importantly, ADCC activity was associated with the modest protective efficacy in the RV144 HIV vaccine trial [6] and has been implicated in phenotypes of viral control [32,33]. Responses characterized by coordinated antibody function, including NK mediated ADCC and NK cytokine secretion/degranulation associated with viral controller phenotypes [34]. ADCC efficiency is linked to specific antibody features including subclass and glycosylation that can be modified to enhance NK cell recruitment and activation. NK cell maturation and education can further enhance HIV-specific ADCC activity [35, 36]. Of note, as with other effector pathways, ADCC is also limited by viral evasion: the viral accessory protein $\mathrm{Vpu}$ antagonizes the antiviral factor tetherin, altering the release of virus aggregates and disabling ADCC mediated viral recognition [37-41]. A small molecule inhibitor of vpu's interaction with tetherin has recently been described suggesting an additional target for intervention [42]. An optimized approach would prime NK cells, induce antibodies with glycan and subclass features maximized for ADCC and could potentially be coupled with pharmacologic blockade of viral immune evasion to further enhance antiviral function.

The collective data highlight the importance of NK cells in HIV disease. There are multiple pathways for target elimination, all balanced by mechanisms of viral evasion that define kinetic windows of maximal response and opportunities for enhancement. In addition to these specific interactions, NK cell recognition of generic signals of stress and infection induced early in HIV infection is an important area for exploration. Optimized NK cell function can be achieved through directly augmenting NK cell function with priming and subset expansion or inhibitory receptor blockade, indirectly through optimization of antibodies to recruit NK cells, and potentially also through blockade of viral accessory proteins to limit immune evasion. 
The Consequences of HIV Infection for NK Cell Distribution and Function

Chronic HIV-1 infection alters the population distribution and functional capacity of NK cells. Numerous studies have described these changes in the setting of HIV infection and have at times produced diverging and contradictory results. This lack of clarity is likely related to multiple factors: the dependence of NK cell function on the associated HLA genotype has not always been fully considered, many studies are cross sectional in nature and may not have adequately controlled for confounders between groups including CMV serostatus, age, and gender, and finally the diversity of NK cell phenotypes is not fully captured in a limited examination of markers [11, $12 \bullet \cdot]$.

NK cells are divided into subsets based on their expression of CD56 and CD16; these subsets are thought to represent stages in NK cell differentiation, although the precise relationship has not been fully defined. Based on immunodeficiency syndromes, both CD $56^{\text {bright }}$ and CD56 ${ }^{\mathrm{dim}}$ populations contribute to antiviral immunity. CD56 ${ }^{\text {bright }} \mathrm{CD} 16^{-}$cells are a minor population in healthy individuals with relatively limited cytotoxic capacity but strong production of cytokines. CD56 ${ }^{\mathrm{dim}} \mathrm{NK}$ cells are the majority of the circulating population with less proliferative potential, increased cytotoxic capacity, and progressive acquisition of KIRs and markers including CD57 during differentiation. A third minor population is the $\mathrm{CD} 6^{\text {neg }} \mathrm{NK}$ cells that are $\mathrm{CD} 16^{+}$; these cells have been difficult to characterize with their limited expression of lineage markers. NK cells express a broad complement of additional receptors including the predominantly inhibitory KIRs; the natural cytotoxicity receptors (NCRs) including NKp30, NKp44, and NKp46; the C-type lectin receptors including NKG2D, NKG2C, and NKG2A; signaling lymphocyte activation (SLAM) family receptors as well as the Fc $\gamma$ receptor CD16 [43]. NK cell degranulation/cytotoxicity and cytokine secretion are governed by the overall balance of activating and inhibitory signals, with an important role for education through inhibitory signaling during development [44]. The roles of each subset in HIV-1 infection are still being defined.

During primary HIV infection, initiation of antiretroviral therapy was associated with reciprocal variation in the $\mathrm{CD} 16^{+} \mathrm{CD} 56^{\mathrm{dim}}$ and $\mathrm{CD} 56^{\text {high }} \mathrm{NK}$ cells, with the latter, more immature population, increasing after therapy [45]. Initiation of therapy also changes immunoregulatory markers; modulation of galectin 9 and $\mathrm{T}$ cell immunoglobulin and mucin-domain containing molecule-3 (TIM-3) in early infection leads to enhanced NK cell activity, but in chronic HIV the interaction may contribute to NK cell dysfunction [46]. Dysregulated TIM-3 expression on NK cells is linked to impaired CD4 recovery after treatment, suggesting that NK cell dysfunction may contribute to a poor immunologic reconstitution in some individuals [47]. $\mathrm{CD} 56^{\text {neg }} \mathrm{CD} 16^{+}$cells with reduced ADCC activity also accumulate during chronic infection, a defect that is significantly restored with viral suppression [48, 49]. Counterbalancing this restoration of NK cell ADCC is the decline in antibody conferred activity after initiation of antiretroviral therapy (ART) [50]. Thus, to fully exploit the potential for NK cell-mediated function in cure/eradication strategies through ADCC, restoration of subset distribution is an essential step, beyond optimizing antibody subclass, glycan structure and titer through vaccination or administration of a monoclonal.

In vitro studies have sought to dissect receptor patterns that lead to NK cell control of HIV infection. When exposed to infected $\mathrm{CD} 4^{+} \mathrm{T}$ cells, autologous NK cells expressing NKG2A responded with CD107a (a marker of degranulation), IFN $\gamma$, and CCL4 at the highest frequencies [51]. Profiling the NKG2A receptor expression in an individual may offer an index of their likelihood to have an effective NK cell response to a therapeutic intervention. In the setting of significant inflammation in a cohort in Uganda with HIV subtype D infection, impaired effector function (IFN $\gamma$ production) and loss of a highly activated subset of NK cells has been described [52]. Further evidence of dysfunction is suggested by studies in the SIV nonhuman primate model demonstrating anergic NK cell accumulation in lymph nodes in chronic infection [53]. Research to redirect or rescue function will be informed by the more complete understanding of the pathology of HIV. An alternative window to identify highly effective activation pathways may be through the enhanced effector capability seen in NK cells from individuals with spontaneous viral control. One recent example is the recent report of a potential role for NK cells in the long-term suppression of HIV-1 in the VISCONTI cohort of posttreatment controllers (Scott-Algara, et al., Abstract 15, CROI 2015, Seattle, WA).

In support of the concept that NK cells can be directed, methods of bolstering NK cell functional capacity in HIV infection have been described. A therapeutic HIV vaccine that restored $\mathrm{CD} 4^{+} \mathrm{T}$ cell IL-2 production led to enhanced production of IFN $\gamma$ by NK cells [54]. Secretion of IFN $\alpha$ by other cells drives NK cell activation and lysis of HIV-infected $\mathrm{CD}^{+}{ }^{+} \mathrm{T}$ cells through NKp46- and NKG2D-mediated signaling [55]. The cross-talk between adaptive and NK cell responses is a critical means for optimizing NK cell function. Coordinating the recruitment and activity of multiple cell types, particularly in the setting of an inflamed and anergic immune system, may be the key to promoting more effective NK cell control of HIV. 


\section{NK Cell Development}

\section{Genetic Determinants of NK Cell Deficiency Syndromes}

Naturally occurring NK cell deficiency syndromes offer important insights into the biology of these cytolytic effectors. Deficiency syndromes are broadly divided into classical NK cell deficiency (CNKD) with numeric and functional deficits, and functional NK cell deficiency (FNKD) in which cells are present but poorly functional (reviewed in [5, 56]). Genetic defects associated with both types of syndrome have been identified. In CNKD1, mutations in the transcription factor GATA2 confer deficits in peripheral NK cell numbers and function, with marked susceptibility to viral infections (varicella zoster virus, herpes simplex virus, cytomegalovirus, human papilloma virus) and mycobacterial infections. These patients specifically lack of the CD56 $6^{\text {bright }}$ subset and show globally impaired expression of NKG2D [57]. A distinct syndrome, CNKD2, is linked to a mutation in MCM4, with a converse phenotype of absence of the CD $56^{\mathrm{dim}}$ population with clinical findings of recurrent viral infections and EBV-driven lymphoproliferative disorders [58]. The deficit in opposite subsets in these two syndromes is striking given their proposed developmental relationship and the susceptibility to viral infections seen in both patient groups. In a separate patient cohort with marked susceptibility to herpesvirus infections and a form of FNKD, studies identified a mutation in the membrane distal domain of CD16. This mutation abrogated an interaction between CD16 and CD2, preventing activation of spontaneous cytotoxicity responses despite preserved ADCC [59]. The efficiency of ADCC responses is also associated with control of herpes simplex virus (HSV). Higher affinity allotypes of CD16 and IgG are linked to control of HSV (versus recurrent outbreaks) and are associated with enhanced ADCC in vitro [60,61]. Multiple additional genetic loci have been linked to deficiencies in NK cell number and function in combined immune deficiency syndromes [62].

Taken together, these findings highlight key functional and developmental aspects of NK cell biology. First, both the $\mathrm{CD} 56^{\text {bright }}$ and $\mathrm{CD} 56^{\mathrm{dim}}$ populations have significant roles in control of virus infections. Second, expression of CD16 has dual significance, promoting cytotoxic activity triggered by $\mathrm{CD} 2$ ligation as well as mediating ADCC. Third, the efficiency of the ADCC response is associated with successful control of herpes virus, reinforcing the role of ADCC in phenotypes of elite control in HIV. These observations may inform some of the disruption seen with shifts in the CD56 expression with chronic HIV infection, and supports the pursuit of both direct antiviral and ADCC strategies in HIV eradication and cure efforts.
Homeostasis and Maturation Signals

Given the defects seen in chronic HIV infection, understanding key differentiation signals in NK cell biology may point to therapeutic strategies to shape or direct their responses. IL-15 is a master regulator of NK cell maturation, survival, and functional competence. IL-15 preferentially expands cytotoxic NK subsets, with less proliferative stimulus for regulatory $\mathrm{T}$ cells than IL-2, shifting the net balance towards activation [63]. Recent human clinical trials in patients with metastatic cancer have demonstrated proliferation and activation of NK cells after administration of recombinant human IL-15 [64••]. In this exploratory study, no objective remissions were achieved; however, there was preliminary evidence of immune activity seen in the clearance of lung lesions in two of the subjects [64.*]. This particular approach is relevant to HIV cure strategies; enhancing innate surveillance by NK cells in concert with latent virus reactivation can circumvent limitations of traditional adaptive responses to HIV. Ongoing efforts to optimize delivery methods (e.g., with heterodimeric formats of the cytokine and a receptor subunit [65]) and synergistic combinations of cytokine with antibody or effector CTLs are active areas of investigation (Euler, Jones, and Alter, in preparation). In a humanized mouse model of HIV infection, use of an IL-15 superagonist (IL-15 bound to the soluble IL-15R $\alpha$ ) effectively blocked HIV infection, in an NK cell dependent manner [66]. In parallel to these studies, multiple clinical trials of adoptive therapy with expanded allogeneic NK cells in cancer are assessing the therapeutic potential of ex vivo expansion and reinfusion into patients [67]. Results of these trials will be critical to define the safety of NK cell expansion and administration and may inform which subsets are critical to immune protection. Both of these approaches, in vivo exposure to IL-15 and ex vivo NK cell manipulation and reinfusion, have potential advantages, and further work is needed to define the most promising avenues.

Multiple additional pathways to modulate NK cell maturation and function are under investigation. Immunomodulatory therapies have potential efficacy in NK cells analogous to the effects described for the adaptive immune system; blocking PD-1 on NK cells from multiple myeloma patients enhanced killing of autologous tumor cells [68]. Indirectly, proteasome inhibitors used in cancer chemotherapy also enhance NK cell function, at least in part by downregulation of HLA molecules triggering missing self responses [69]. Due to their highly potent cytotoxic activity, an array of inhibitory receptors tonically repress the function of NK cells [44], offering another target for intervention. Direct blockade of inhibitory KIR receptors 
has been shown to augment spontaneous NK cell cytotoxicity in animal models [70] and is in exploratory clinical trials of cancer therapy [71].

\section{NK Cell Activation and Specificity}

Efforts to modulate NK cells with cytokines and direct therapies point back to a critical question in NK cell biology. While there is considerable variation in NK cell response potential, the precise determinants (e.g., receptor expression and maturation state) of an NK cell response to pathogen challenge are not clear. In contrast to adaptive immune responses that are governed by antigen-specific gene-rearranged receptors, NK cells integrate multiple activating and inhibitory signals to reach a threshold of activation. Importantly, both the KIR genotype of an individual [22] and the multiple unique combinations of receptors that can be expressed $[11,12 \bullet \cdot]$ contribute to the NK population diversity of receptor expression and activation potential. However, apart from the genetic determinants, environmental and stochastic factors including infectious exposures such as $\mathrm{CMV}$ are also capable of profoundly shaping the response potential of NK cells through alterations in repertoire distribution [72, 73]. Building on differences in surface receptors, work defining transcriptional signatures of NK cells in distinct activation states has provided a context to understand NK cell activation alone and in relationship to innate lymphoid cells and traditional T cells [74]. Moving forward, to truly harness NK cells towards a therapeutic goal, the determinants of NK cell repertoire composition, activation threshold, and role in susceptibility to infection must be defined.

\section{NK Cell Repertoire Diversity}

Detailed studies with high dimensional analysis enabled by cytometry by time of flight (CyTOF) has provided novel insights into the diversity and specificity of the NK cell repertoire $[11,12 \bullet \cdot]$. These studies reveal the potential for NK cell combinatorial receptor diversity, with estimates of up to 30 , 000 unique receptor phenotypes on a single NK cell in an individual subject. Remarkably, most individual receptor combinations contributed to only a minority of the overall $\mathrm{NK}$ cell population, with only the less mature $\mathrm{NKG}_{2} \mathrm{~A}^{+} /$ $\mathrm{CD} 94^{+} \mathrm{NK}$ cells and more mature $\mathrm{CD} 57^{+} / \mathrm{CD} 16^{+}$population as primary distinct phenotypes [11]. Distinct responses to stimuli, proliferative potential, cytokine production, and cytolytic activity are seen in subsets defined by the expression of CD57 [75]. Diversity of phenotypes in adults is correlated with CD57 expression, suggesting that maturation leads to divergence, and was associated with risk of HIV acquisition. In case control analyses of high-risk women, increased NK cell diversity was associated with an increased probability of HIV-1 infection [12••]. It is not clear whether the NK diversity reflects a "distraction" from the challenging pathogen that increases susceptibility or if it is linked to other immune characteristics such as $T$ cell exhaustion. These data raise questions about preventative and therapeutic vaccination goals; a naïve repertoire may have more flexibility and protective potential than one directed at other pathogens; however, a vaccine-directed, pathogen-specific response should be superior to both. Understanding how clonal NK cell populations can be reshaped and directed will critically inform the potential to harness a range of biological functions of NK cells through vaccine design.

\section{NK Cell Memory}

While classically considered as nonspecific innate effector cells, there is growing data to support adaptive, memory-like features of NK cells. These features can be divided into two categories, antigen-specific responses with classical recall characteristics and a more general alteration in effector function conditioned by an infection, vaccination, or inflammatory environment in line with the phenomenon of innate training in the myeloid compartment $[14,76]$. Both types of memory responses are potential targets for immune intervention.

The mouse model of hapten-mediated contact hypersensitivity reactions provided the first demonstration of persistent, antigen-specific, and transferrable memory in NK cells [77]. Further work identified the importance of the chemokine receptor CXCR6 in memory development and extended the observations from haptens to influenza virus-like particles and inactivated vaccinia virus; the receptors mediating this recognition have remained undefined [78]. The first report of NK cell memory in the setting of a viral infection demonstrated NK cell-mediated responses to mouse cytomegalovirus (MCMV) [79]. Development of murine CMV (MCMV) memory was linked to interactions between the NK receptor LY49H and the viral glycoprotein $\mathrm{m} 157$ [80, 81], and memory cells were broadly distributed in the tissues, in contrast to the liver-resident hapten-directed memory cells [79]. More recently, memory NK cells specific for SIV antigens have been identified in the nonhuman primate model [13••]. NK cells derived from rhesus macaques infected with SIV or a virus combining SIV and HIV (SHIV) showed antigen-specific killing of targets pulsed with HIV protein components, and a persistent response to vaccine antigens was identified in animals who had received an adenovirus 26 (Ad26) vectored SIV vaccine 5 years earlier [13*0]. These memory NK cells were elicited by both vaccination and infection, were durable and specific, and were recovered from both the liver and spleen [13••]. These animal model data demonstrate antigen-specific NK cell memory and while direct evidence in humans is lacking, these studies strongly support efforts to identify and harness this activity in human disease. Access to the relevant cell populations, e.g., hepatic or splenic cells, may be critical to identify 
these responses and should be one focus of study. In parallel, in vitro studies to induce memory-like response and animal models including the humanized mouse offer additional opportunities to explore the biology of these cytolytic effectors.

Antigen-specific memory in NK cells has clear parallels to traditional adaptive immunological memory. Consistent with their unique role bridging the innate and adaptive responses, NK cells are also capable of a memory phenotype that is induced by more generalized signals. Exposure of murine NK cells to cytokines (IL-12, IL-15, and IL-18) in vitro can induce a memory-like phenotype. When transferred into naïve hosts, these cells have a resting phenotype, but produce more IFN $\gamma$ on cytokine restimulation, a response that remains detectable 3 weeks after cell transfer [82]. Human NK cells have similar properties, with a brief exposure to cytokines conditioning enhanced responsiveness on rechallenge [83]. How this memory-like state is established is a topic of investigation, but epigenetic remodeling of a noncoding sequence at the IFNG gene linked to increased IFN production can be induced by in vitro cytokine priming [84]. Epigenetic programming also underlies the sustained shifts in NK cell profiles that are seen in human CMV (HCMV) infection. HCMV infection drives expansion of a population of CD94-NKG2C NK cells [85], and in the setting of hematopoietic stem cell transplantation, this population has been demonstrated to have a memory-like response to CMV [86]. The interaction between HLA-E and CD94-NKG2C contributes to this expansion [87], but additional pathways to NK memory in HCMV are also operative, as evidenced by the FceRI $\gamma$-deficient adaptive NK cells that expand after activation through CD16 [16••]. HCMV infection is associated with sustained changes in NK

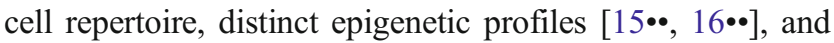
altered functional profiles $[15 \bullet \bullet, 16 \bullet \cdot$. The responses appear to be directed by exposure to the pathogen, in some cases directly through a viral antigen, and in other cases through secondary recognition of specific antibody. In congruence with the findings in HCMV, an NK cell population that lacks $\mathrm{FcR} \gamma$ expression and has enhanced ADCC activity was identified in HIV-infected subjects, with some features shared with the memory-like population induced by CMV and some unique surface receptor characteristics [88]. These findings have compelling links to both the biology of adaptive immune responses and the growing field of research in innate training and tolerance whereby epigenetic programs direct altered secondary responses after an initial exposure [14, 89], and both pathways can be harnessed towards goals of HIV prevention and cure.

\section{NK Cell Editing of Adaptive Immunity}

Recent studies have focused attention on a critical role for NK cells in the shaping adaptive immune responses. In MCMV infection, NK cells rapidly eliminate infected targets, limiting the type I interferon response, preserving conventional dendritic cells and CD8 T cell responses [90]. In this infection, NK cells limit exposure of CD4 and CD8 T cells to infected dendritic cells shaping the subsequent adaptive response [91] and importantly, also limit tissue site $\mathrm{T}$ cell-mediated pathology [92]. In HIV, NK cell editing of dendritic cells is aberrant in the context of chronic inflammation and elevated IL-10, leading to poorly dendritic cells with limited immunogenicity [93]. Similarly, NK cells dictate immune response characteristics in an indirect fashion in the lymphocytic choriomeningitis virus (LCMV) mouse model. In this system, NK cells have no significant role in elimination of virusinfected targets, but they eliminate activated CD4 T cells either limiting immunopathology, or contributing to exhaustion and inefficient CD8 $\mathrm{T}$ cell control in chronic infection [94-96]. NK cells have also been shown to shape the induction of antibody; perforin-mediated elimination of $\mathrm{T}$ follicular helper (Tfh) cells in the lymph node by NK cells in acute infection was shown to disrupt germinal center formation, limiting immune memory development [97••]. Tfh cells have been identified as the dominant population supporting replication and virus production in viremic HIV-1 infection [98], and are likely a significant contributor to the HIV-1 reservoir.

The context-dependent effects of NK cells on adaptive immunity highlight the need for careful direction of efforts to harness their activity in HIV infection. Specifically, disabling their CD4 suppressive effects after vaccination may promote more breadth of antibody response. In contrast, during acute infection, enhancing NK-mediated elimination of Tfh cells may limit the size of the reservoir that is established. Likewise, during a curative intervention, unleashing NK cell targeting of Tfh cells could again lead to reservoir reduction. Directed recruitment of NK cells to lymph nodes also offers a pathway to increase their efficacy [99]. The impact of NK cells on interventions targeting adaptive responses offers a novel pathway to enhance efforts at prevention and cure [3].

\section{Conclusions}

NK cells have emerged as multifunctional effector cells with the potential to control infections and shape adaptive immune responses. NK cells exert immune pressure on HIV and contribute to protective vaccine responses and some phenotypes of immune control. Significantly, there has been little effort to optimize, direct, or specifically target NK cells in therapeutic and preventative interventions. Mounting evidence from basic biological studies of NK cells points to the multiple avenues to enhance their antiviral and immunomodulatory function in HIV. The barrier to eliciting effective immunity to HIV is significant, and the potential to harness this important and potent effector population must be a high-priority component of research efforts in prevention and cure. 
Acknowledgments We would like to thank Avery Normandin for expert assistance in figure design.

\section{Compliance with Ethical Standards}

Conflict of Interest Eileen Scully and Galit Alter declare that they have no conflict of interest.

Human and Animal Rights and Informed Consent This article does not contain any studies with human or animal subjects performed by any of the authors.

Open Access This article is distributed under the terms of the Creative Commons Attribution 4.0 International License (http:// creativecommons.org/licenses/by/4.0/), which permits unrestricted use, distribution, and reproduction in any medium, provided you give appropriate credit to the original author(s) and the source, provide a link to the Creative Commons license, and indicate if changes were made.

\section{References}

Papers of particular interest, published recently, have been highlighted as:

-• Of major importance

1. Biron CA, Byron KS, Sullivan JL. Severe herpesvirus infections in an adolescent without natural killer cells. N Engl J Med. 1989;320: 1731-5.

2. Fleisher G, Starr S, Koven N, Kamiya H, Douglas SD, Henle W. A non-x-linked syndrome with susceptibility to severe Epstein-Barr virus infections. J Pediatr. 1982;100:727-30.

3. Rydyznski CE, Waggoner SN. Boosting vaccine efficacy the natural (killer) way. Trends Immunol. 2015;36:536-46.

4. O'Sullivan TE, Sun JC, Lanier LL. Natural killer cell memory. Immunity. 2015;43:634-45.

5. Orange JS. Natural killer cell deficiency. J Allergy Clin Immunol. 2013;132:515-25. quiz 526.

6. Haynes BF, Gilbert PB, McElrath MJ, et al. Immune-correlates analysis of an HIV-1 vaccine efficacy trial. N Engl J Med. 2012;366:1275-86.

7. Chung AW, Ghebremichael M, Robinson H, et al. Polyfunctional Fc-effector profiles mediated by IgG subclass selection distinguish RV144 and VAX003 vaccines. Sci Transl Med. 2014;6:228ra238.

8. Bournazos S, Klein F, Pietzsch J, Seaman MS, Nussenzweig MC, Ravetch JV. Broadly neutralizing anti-HIV-1 antibodies require Fc effector functions for in vivo activity. Cell. 2014;158:1243-53.

9. Chung AW, Crispin M, Pritchard L, et al. Identification of antibody glycosylation structures that predict monoclonal antibody Fceffector function. AIDS. 2014;28:2523-30.

10. Ackerman ME, Alter G. Opportunities to exploit non-neutralizing HIV-specific antibody activity. Curr HIV Res. 2013;11:365-77.

11. Horowitz A, Strauss-Albee DM, Leipold M, et al. Genetic and environmental determinants of human NK cell diversity revealed by mass cytometry. Sci Transl Med. 2013;5:208ra145.

12.• Strauss-Albee DM, Fukuyama J, Liang EC, et al. Human NK cell repertoire diversity reflects immune experience and correlates with viral susceptibility. Sci Transl Med. 2015;7:297ra115. Using high dimensional analysis, this study shows the potential diversity of unique NK cell phenotypes determined by different receptor combinations and links this diversity to susceptibility to infection with HIV.

13.• Reeves RK, Li H, Jost S, et al. Antigen-specific NK cell memory in rhesus macaques. Nat Immunol. 2015;16:927-32. This study demonstrates that antigen specific memory to SIV and SHIV antigens can be generated by infection and vaccination in nonhuman primates.

14. Netea MG, Latz E, Mills KH, O’Neill LA. Innate immune memory: a paradigm shift in understanding host defense. Nat Immunol. 2015;16:675-9.

15.• Schlums H, Cichocki F, Tesi B, et al. Cytomegalovirus infection drives adaptive epigenetic diversification of NK cells with altered signaling and effector function. Immunity. 2015;42:443-56. This study is paired with the work of Lee et al., and demonstrates that CMV infection can induce epigenetic programs associated with altered functional profiles of NK cells.

16.• Lee J, Zhang T, Hwang I, et al. Epigenetic modification and antibody-dependent expansion of memory-like NK cells in human cytomegalovirus-infected individuals. Immunity. 2015;42:431-42. Paired with the work of Schlums et al., this study provides evidence for epigenetic changes in NK cells that mediate functional programming and demonstrates the role of antibody in shaping theses responses.

17. Khakoo SI, Thio CL, Martin MP, et al. HLA and NK cell inhibitory receptor genes in resolving hepatitis $\mathrm{C}$ virus infection. Science. 2004;305:872-4.

18. Martin MP, Gao X, Lee JH, et al. Epistatic interaction between KIR3DS1 and HLA-B delays the progression to AIDS. Nat Genet. 2002;31:429-34.

19. Martin MP, Qi Y, Gao X, et al. Innate partnership of HLA-B and KIR3DL1 subtypes against HIV-1. Nat Genet. 2007;39:733-40.

20. Boulet S, Kleyman M, Kim JY, et al. A combined genotype of KIR3DL1 high expressing alleles and HLA-B*57 is associated with a reduced risk of HIV infection. AIDS. 2008;22:1487-91.

21. Boulet S, Sharafi S, Simic N, et al. Increased proportion of KIR3DS1 homozygotes in HIV-exposed uninfected individuals. AIDS. 2008;22:595-9.

22. Long EO, Kim HS, Liu D, Peterson ME, Rajagopalan S. Controlling natural killer cell responses: integration of signals for activation and inhibition. Annu Rev Immunol. 2013;31:227-58.

23. Alter G, Heckerman D, Schneidewind A, et al. HIV-1 adaptation to NK-cell-mediated immune pressure. Nature. 2011;476:96-100.

24. Alter G, Martin MP, Teigen N, et al. Differential natural killer cellmediated inhibition of HIV-1 replication based on distinct KIR/ HLA subtypes. J Exp Med. 2007;204:3027-36.

25. Holzemer A, Thobakgale CF, Jimenez Cruz CA, et al. Selection of an HLA-C*03:04-Restricted HIV-1 p24 gag sequence variant is associated with viral escape from KIR2DL3+ natural killer cells: data from an observational cohort in South Africa. PLoS Med. 2015;12:e1001900.

26. van Teijlingen NH, Holzemer A, Korner C, et al. Sequence variations in HIV-1 p24 Gag-derived epitopes can alter binding of KIR2DL2 to HLA-C*03:04 and modulate primary natural killer cell function. AIDS. 2014;28:1399-408.

27. Collins KL, Chen BK, Kalams SA, Walker BD, Baltimore D. HIV1 Nef protein protects infected primary cells against killing by cytotoxic T lymphocytes. Nature. 1998;391:397-401.

28. Specht A, DeGottardi MQ, Schindler M, Hahn B, Evans DT, Kirchhoff F. Selective downmodulation of HLA-A and -B by Nef alleles from different groups of primate lentiviruses. Virology. 2008;373:229-37.

29. Norman JM, Mashiba M, McNamara LA, et al. The antiviral factor APOBEC3G enhances the recognition of HIV-infected primary T cells by natural killer cells. Nat Immunol. 2011;12:975-83.

30. Richard J, Sindhu S, Pham TN, Belzile JP, Cohen EA. HIV-1 Vpr up-regulates expression of ligands for the activating NKG2D 
receptor and promotes NK cell-mediated killing. Blood. 2010;115: 1354-63.

31. Shah AH, Sowrirajan B, Davis ZB, et al. Degranulation of natural killer cells following interaction with HIV-1-infected cells is hindered by downmodulation of NTB-A by Vpu. Cell Host Microbe. 2010;8:397-409.

32. Wren LH, Chung AW, Isitman G, et al. Specific antibodydependent cellular cytotoxicity responses associated with slow progression of HIV infection. Immunology. 2013;138:116-23.

33. Lambotte $\mathrm{O}$, Ferrari $\mathrm{G}$, Moog $\mathrm{C}$, et al. Heterogeneous neutralizing antibody and antibody-dependent cell cytotoxicity responses in HIV-1 elite controllers. AIDS. 2009;23:897-906.

34. Ackerman ME, Mikhailova A, Brown EP, et al. Polyfunctional HIV-Specific Antibody Responses Are Associated with Spontaneous HIV Control. PLoS Pathog. 2016;12:e1005315.

35. Parsons, M.S., Loh, L., Gooneratne, S., Center, R.J., and Kent, S.J. Role of education and differentiation in determining the potential of natural killer cells to respond to antibody-dependent stimulation. AIDS. 2014;28:2781-6

36. Gooneratne SL, Richard J, Lee WS, Finzi A, Kent SJ, Parsons MS. Slaying the Trojan horse: natural killer cells exhibit robust antiHIV-1 antibody-dependent activation and cytolysis against allogeneic T cells. J Virol. 2015;89:97-109.

37. Alvarez RA, Hamlin RE, Monroe A, et al. HIV-1 Vpu antagonism of tetherin inhibits antibody-dependent cellular cytotoxic responses by natural killer cells. J Virol. 2014;88:6031-46.

38. Arias JF, Heyer LN, von Bredow B, et al. Tetherin antagonism by Vpu protects HIV-infected cells from antibody-dependent cell-mediated cytotoxicity. Proc Natl Acad Sci U S A. 2014;111:6425-30.

39. Kramski M, Stratov I, Kent SJ. The role of HIV-specific antibodydependent cellular cytotoxicity in HIV prevention and the influence of the HIV-1 Vpu protein. AIDS. 2015;29:137-44.

40. Li SX, Barrett BS, Heilman KJ, et al. Tetherin promotes the innate and adaptive cell-mediated immune response against retrovirus infection in vivo. J Immunol. 2014;193:306-16.

41. Pham TN, Lukhele S, Hajjar F, Routy JP, Cohen EA. HIV Nef and Vpu protect HIV-infected CD4+ T cells from antibody-mediated cell lysis through down-modulation of CD4 and BST2. Retrovirology. 2014;11:15.

42. Mi Z, Ding J, Zhang Q, et al. A small molecule compound IMB-LA inhibits HIV-1 infection by preventing viral Vpu from antagonizing the host restriction factor BST-2. Sci Rep. 2015;5:18499.

43. Martinet L, Smyth MJ. Balancing natural killer cell activation through paired receptors. Nat Rev Immunol. 2015;15:243-54.

44. Sun JC, Lanier LL. NK cell development, homeostasis and function: parallels with CD8(+) T cells. Nat Rev Immunol. 2011;11: 645-57.

45. Ripa M, Pogliaghi M, Chiappetta S, et al. Dynamics of adaptive and innate immunity in patients treated during primary human immunodeficiency virus infection: results from Maraviroc in HIV Acute Infection (MAIN) randomized clinical trial. Clin Microbiol Infect. 2015;21(876):e871-874.

46. Jost S, Moreno-Nieves UY, Garcia-Beltran WF, et al. Dysregulated Tim-3 expression on natural killer cells is associated with increased Galectin-9 levels in HIV-1 infection. Retrovirology. 2013;10:74.

47. de Kivit S, Lempsink LJ, Plants J, Martinson J, Keshavarzian A, Landay AL. Modulation of TIM-3 expression on NK and T cell subsets in HIV immunological non-responders. Clin Immunol. 2015;156:28-35.

48. Mavilio D, Lombardo G, Benjamin J, et al. Characterization of CD56-/CD16+ natural killer (NK) cells: a highly dysfunctional NK subset expanded in HIV-infected viremic individuals. Proc Natl Acad Sci U S A. 2005;102:2886-91.

49. Brunetta E, Hudspeth KL, Mavilio D. Pathologic natural killer cell subset redistribution in HIV-1 infection: new insights in pathophysiology and clinical outcomes. J Leukoc Biol. 2010;88:1119-30.
50. Madhavi, V., Ana-Sosa-Batiz, F.E., Jegaskanda, S., Center, R.J., Winnall, W.R., Parsons, M.S., Ananworanich, J., Cooper, D.A., Kelleher, A.D., Hsu, D., et al. Antibody-dependent effector functions against HIV decline in subjects receiving antiretroviral therapy. J Infect Dis. 2015;211:529-38.

51. Lisovsky I, Isitman G, Song R, et al. A higher frequency of $\mathrm{NKG} 2 \mathrm{~A}+$ than of NKG2A- NK cells responds to autologous HIV-infected CD4 cells irrespective of whether or not they coexpress KIR3DL1. J Virol. 2015;89:9909-19.

52. Naluyima P, Eller MA, Laeyendecker O, et al. Impaired natural killer cell responses are associated with loss of the highly activated NKG2A(+)CD57(+)CD56(dim) subset in HIV-1 subtype D infection in Uganda. AIDS. 2014;28:1273-8.

53. Schafer JL, Li H, Evans TI, Estes JD, Reeves RK. Accumulation of cytotoxic CD16+ NK cells in simian immunodeficiency virusinfected lymph nodes associated with in situ differentiation and functional anergy. J Virol. 2015;89:6887-94.

54. Jost S, Tomezsko PJ, Rands K, et al. CD4+ T-cell help enhances NK cell function following therapeutic HIV-1 vaccination. J Virol. 2014;88:8349-54.

55. Tomescu C, Mavilio D, Montaner LJ. Lysis of HIV-1-infected autologous CD4+ primary $\mathrm{T}$ cells by interferon-alpha-activated NK cells requires NKp46 and NKG2D. AIDS. 2015;29:1767-73.

56. Wood SM, Ljunggren HG, Bryceson YT. Insights into NK cell biology from human genetics and disease associations. Cell Mol Life Sci. 2011;68:3479-93.

57. Mace EM, Hsu AP, Monaco-Shawver L, et al. Mutations in GATA2 cause human NK cell deficiency with specific loss of the CD56(bright) subset. Blood. 2013;121:2669-77.

58. Gineau L, Cognet C, Kara N, et al. Partial MCM4 deficiency in patients with growth retardation, adrenal insufficiency, and natural killer cell deficiency. J Clin Invest. 2012;122:821-32.

59. Grier JT, Forbes LR, Monaco-Shawver L, et al. Human immunodeficiency-causing mutation defines CD16 in spontaneous NK cell cytotoxicity. J Clin Invest. 2012;122:3769-80.

60. Moraru M, Cisneros E, Gomez-Lozano N, et al. Host genetic factors in susceptibility to herpes simplex type 1 virus infection: contribution of polymorphic genes at the interface of innate and adaptive immunity. J Immunol. 2012;188:4412-20.

61. Moraru M, Black LE, Muntasell A, et al. NK cell and Ig interplay in defense against Herpes Simplex Virus Type 1: epistatic interaction of CD16A and $\operatorname{IgG1}$ allotypes of variable affinities modulates antibody-dependent cellular cytotoxicity and susceptibility to clinical reactivation. J Immunol. 2015;195:1676-84.

62. Voss, M., and Bryceson, Y.T. Natural killer cell biology illuminated by primary immunodeficiency syndromes in humans. Clin Immunol. 2015.

63. Berger C, Berger M, Hackman RC, et al. Safety and immunologic effects of IL-15 administration in nonhuman primates. Blood. 2009;114:2417-26.

64.•Conlon KC, Lugli E, Welles HC, et al. Redistribution, hyperproliferation, activation of natural killer cells and CD8 T cells, and cytokine production during first-in-human clinical trial of recombinant human interleukin-15 in patients with cancer. J Clin Oncol. 2015;33:74-82. This provides first-in-human demonstration of the safety and effects of in vivo administration of IL-15 on NK cell dynamics.

65. Bergamaschi C, Kulkarni V, Rosati M, et al. Intramuscular delivery of heterodimeric IL-15 DNA in macaques produces systemic levels of bioactive cytokine inducing proliferation of $\mathrm{NK}$ and $\mathrm{T}$ cells. Gene Ther. 2015;22:76-86.

66. Seay $\mathrm{K}$, Church $\mathrm{C}$, Zheng JH, et al. In vivo activation of human NK cells by treatment with an interleukin- 15 superagonist potently inhibits acute in vivo HIV-1 infection in humanized mice. J Virol. 2015;89:6264-74. 
67. Lim O, Jung MY, Hwang YK, Shin EC. Present and future of allogeneic natural killer cell therapy. Front Immunol. 2015;6:286.

68. Benson Jr DM, Bakan CE, Mishra A, et al. The PD-1/PD-L1 axis modulates the natural killer cell versus multiple myeloma effect: a therapeutic target for CT-011, a novel monoclonal anti-PD-1 antibody. Blood. 2010;116:2286-94.

69. Wu X, Shao Y, Tao Y, et al. Proteasome inhibitor lactacystin augments natural killer cell cytotoxicity of myeloma via downregulation of HLA class I. Biochem Biophys Res Commun. 2011;415: 187-92.

70. Kohrt HE, Thielens A, Marabelle A, et al. Anti-KIR antibody enhancement of anti-lymphoma activity of natural killer cells as monotherapy and in combination with anti-CD20 antibodies. Blood. 2014;123:678-86.

71. Childs RW, Carlsten M. Therapeutic approaches to enhance natural killer cell cytotoxicity against cancer: the force awakens. Nat Rev Drug Discov. 2015;14:487-98.

72. Beziat V, Liu LL, Malmberg JA, et al. NK cell responses to cytomegalovirus infection lead to stable imprints in the human KIR repertoire and involve activating KIRs. Blood. 2013;121:2678-88.

73. Lopez-Verges S, Milush JM, Schwartz BS, et al. Expansion of a unique CD57(+)NKG2Chi natural killer cell subset during acute human cytomegalovirus infection. Proc Natl Acad Sci U S A. 2011;108:14725-32.

74. Bezman NA, Kim CC, Sun JC, et al. Molecular definition of the identity and activation of natural killer cells. Nat Immunol. 2012;13:1000-9.

75. Nielsen CM, White MJ, Goodier MR, Riley EM. Functional significance of CD57 expression on human NK cells and relevance to disease. Front Immunol. 2013;4:422.

76. Cerwenka, A., and Lanier, L.L. Natural killer cell memory in infection, inflammation and cancer. Nat Rev Immunol. 2016.

77. O'Leary JG, Goodarzi M, Drayton DL, von Andrian UH. T celland $\mathrm{B}$ cell-independent adaptive immunity mediated by natural killer cells. Nat Immunol. 2006;7:507-16.

78. Paust S, Gill HS, Wang BZ, et al. Critical role for the chemokine receptor CXCR6 in NK cell-mediated antigen-specific memory of haptens and viruses. Nat Immunol. 2010;11:1127-35.

79. Sun JC, Beilke JN, Lanier LL. Adaptive immune features of natural killer cells. Nature. 2009;457:557-61.

80. Arase H, Mocarski ES, Campbell AE, Hill AB, Lanier LL. Direct recognition of cytomegalovirus by activating and inhibitory NK cell receptors. Science. 2002;296:1323-6.

81. Smith HR, Heusel JW, Mehta IK, et al. Recognition of a virusencoded ligand by a natural killer cell activation receptor. Proc Natl Acad Sci U S A. 2002;99:8826-31.

82. Cooper MA, Elliott JM, Keyel PA, Yang L, Carrero JA, Yokoyama WM. Cytokine-induced memory-like natural killer cells. Proc Natl Acad Sci U S A. 2009;106:1915-9.

83. Romee R, Schneider SE, Leong JW, et al. Cytokine activation induces human memory-like NK cells. Blood. 2012;120:4751-60.
84. Luetke-Eversloh M, Hammer Q, Durek P, et al. Human cytomegalovirus drives epigenetic imprinting of the IFNG locus in NKG2Chi natural killer cells. PLoS Pathog. 2014;10:e1004441.

85. Guma M, Angulo A, Vilches C, Gomez-Lozano N, Malats N, Lopez-Botet M. Imprint of human cytomegalovirus infection on the NK cell receptor repertoire. Blood. 2004;104:3664-71.

86. Foley B, Cooley S, Verneris MR, et al. Human cytomegalovirus $(\mathrm{CMV})$-induced memory-like $\mathrm{NKG} 2 \mathrm{C}(+) \mathrm{NK}$ cells are transplantable and expand in vivo in response to recipient CMV antigen. $\mathrm{J}$ Immunol. 2012;189:5082-8.

87. Rolle A, Pollmann J, Ewen EM, et al. IL-12-producing monocytes and HLA-E control HCMV-driven NKG2C+ NK cell expansion. J Clin Invest. 2014;124:5305-16.

88. Zhou J, Amran FS, Kramski M, et al. An NK cell population lacking FcRgamma is expanded in chronically infected HIV patients. $\mathrm{J}$ Immunol. 2015;194:4688-97.

89. Kleinnijenhuis J, Quintin J, Preijers F, et al. BCG-induced trained immunity in NK cells: role for non-specific protection to infection. Clin Immunol. 2014;155:213-9.

90. Robbins $\mathrm{SH}$, Bessou G, Cornillon A, et al. Natural killer cells promote early CD8 T cell responses against cytomegalovirus. PLoS Pathog. 2007;3:e123.

91. Andrews DM, Estcourt MJ, Andoniou CE, et al. Innate immunity defines the capacity of antiviral T cells to limit persistent infection. $\mathrm{J}$ Exp Med. 2010;207:1333-43.

92. Schuster IS, Wikstrom ME, Brizard G, et al. TRAIL+ NK cells control CD4+ T cell responses during chronic viral infection to limit autoimmunity. Immunity. 2014;41:646-56.

93. Alter G, Kavanagh D, Rihn S, et al. IL-10 induces aberrant deletion of dendritic cells by natural killer cells in the context of HIV infection. J Clin Invest. 2010;120:1905-13.

94. Lang PA, Lang KS, Xu HC, et al. Natural killer cell activation enhances immune pathology and promotes chronic infection by limiting CD8+ T-cell immunity. Proc Natl Acad Sci U S A. 2012;109:1210-5.

95. Waggoner SN, Cornberg M, Selin LK, Welsh RM. Natural killer cells act as rheostats modulating antiviral T cells. Nature. 2012;481: 394-8.

96. Waggoner SN, Daniels KA, Welsh RM. Therapeutic depletion of natural killer cells controls persistent infection. J Virol. 2014;88: 1953-60.

97.• Rydyznski C, Daniels KA, Karmele EP, et al. Generation of cellular immune memory and B-cell immunity is impaired by natural killer cells. Nat Commun. 2015;6:6375. This study adds to the data on NK cell editing of $T$ cell responses demonstrating the role of $N K$ cells in shaping the development of immunologic memory through antibody generation.

98. Perreau M, Savoye AL, De Crignis E, et al. Follicular helper T cells serve as the major CD4 T cell compartment for HIV-1 infection, replication, and production. J Exp Med. 2013;210:143-56.

99. Luteijn R, Sciaranghella G, van Lunzen J, et al. Early viral replication in lymph nodes provides HIV with a means by which to escape NK-cell-mediated control. Eur J Immunol. 2011;41:2729-40. 